\title{
Different processes shape prokaryotic and picoeukaryotic assemblages in the sunlit ocean microbiome
}

Ramiro Logares ${ }^{1,2^{*}}$, Ina M. Deutschmann ${ }^{1}$, Caterina. R. Giner ${ }^{1}$, Anders K. Krabberød ${ }^{2}$, Thomas S. B. Schmidt ${ }^{3}$, Laura Rubinat-Ripoll ${ }^{4}$, Mireia Mestre ${ }^{1}$, Guillem Salazar ${ }^{5}$, Clara Ruiz-González ${ }^{1}$, Marta Sebastián ${ }^{1,6}$, Colomban de Vargas ${ }^{4}$, Silvia G. Acinas ${ }^{1}$, Carlos M. Duarte $^{7}$, Josep M. Gasol ${ }^{1,8}$, Ramon Massana ${ }^{1}$

${ }^{1}$ Institute of Marine Sciences (ICM), CSIC, 08003, Barcelona, Catalonia, Spain

${ }^{2}$ Section for Genetics and Evolutionary Biology, Department of Biosciences, University of Oslo, N-0316 Oslo, Norway

${ }^{3}$ European Molecular Biology Laboratory, Meyerhofstr. 1, D-69117, Heidelberg, Germany

${ }^{4}$ Sorbonne Universités, UPMC Univ Paris 06, CNRS UMR 7144, Adaptation et Diversité en Milieu Marin, Equipe EPEP, Station Biologique de Roscoff, 29680 Roscoff, France

${ }^{5}$ ETH Zurich, Institute of Microbiology, 8093 Zurich, Switzerland

${ }^{6}$ Oceanography and Global Change Institute, IOCAG, University of Las Palmas de Gran Canaria, ULPGC, Gran Canaria 35214, Spain.

${ }^{7}$ King Abdullah University of Science and Technology (KAUST), Red Sea Research Center (RSRC), Thuwal, Saudi Arabia

${ }^{8}$ Centre for Marine Ecosystems Research, School of Science, Edith Cowan University, Joondalup, WA, Australia

\section{* Corresponding author:}

\section{Ramiro Logares}

Institute of Marine Sciences (ICM), CSIC,

Passeig Marítim de la Barceloneta, 37-49,

08003, Barcelona, Catalonia, Spain

e-mail: ramiro.logares@gmail.com

Tel: +34 93 2309500; Fax: +34 932309555

Keywords: microbiome, ocean, structure, picoeukaryotes, prokaryotes 


\section{ABSTRACT}

The smallest members of the sunlit-ocean microbiome (prokaryotes and

48 picoeukaryotes) participate in a plethora of ecosystem functions with planetary-scale

49 effects. Understanding the processes determining the spatial turnover of this assemblage

50 can help us better comprehend the links between microbiome species composition and

51 ecosystem function. Ecological theory predicts that selection, dispersal and drift are

52 main drivers of species distributions, yet, the relative quantitative importance of these

53 ecological processes in structuring the surface-ocean microbiome is barely known. Here

54 we quantified the role of selection, dispersal and drift in structuring surface-ocean

55 prokaryotic and picoeukaryotic assemblages by using community DNA-sequence data

56 collected during the global Malaspina expedition. We found that dispersal limitation

57 was the dominant process structuring picoeukaryotic communities, while a balanced

58 combination of dispersal limitation, selection and drift shaped prokaryotic counterparts.

59 Subsequently, we determined the agents exerting abiotic selection as well as the spatial

60 patterns emerging from the action of different ecological processes. We found that

61 selection exerted via temperature had a strong influence on the structure of prokaryotic

62 communities, particularly on species co-occurrences, a pattern not observed among

63 communities of picoeukaryotes. Other measured abiotic variables had limited selective

64 effects on microbiome structure. Picoeukaryotes presented a higher differentiation

65 between neighbouring communities and a higher distance-decay when compared to

66 prokaryotes, agreeing with their higher dispersal limitation. Finally, drift seemed to

67 have a limited role in structuring the sunlit-ocean microbiome. The different

68 predominance of ecological processes acting on particular subsets of the ocean microbiome suggests uneven responses to environmental change. 


\section{SIGNIFICANCE STATEMENT}

72

The global ocean contains one of the largest microbiomes on Earth and changes

73 on its structure can impact the functioning of the biosphere. Yet, we are far from

74 understanding the mechanisms that structure the global ocean microbiome, that is, the

75 relative importance of environmental selection, dispersal and random events (drift). We

76 evaluated the role of these processes at the global scale, based on data derived from a

77 circumglobal expedition and found that these ecological processes act differently on

78 prokaryotes and picoeukaryotes, two of the main components of the ocean microbiome.

79 Our work represents a significant contribution to understand the assembly of marine

80 microbial communities, providing also insights on the links between ecological

81 mechanisms, microbiome structure and ecosystem function.

82 
INTRODUCTION

The surface ocean microbiome is a pivotal underpinning of global

biogeochemical cycles, thus being crucial for the functioning of the biosphere (1-4).

The smallest ocean microbes, the picoplankton, have a key role in the global carbon

87 cycle (4), being responsible for an important fraction of the total atmospheric carbon and nitrogen fixation in the ocean (5-7), which supports $\sim 46 \%$ of the global primary productivity (8). Oceanic picoplankton plays a fundamental role in processing organic matter by recycling nutrients and carbon to support additional production as well as by channelling organic carbon to upper trophic levels through food webs $(4,5,9)$. The ocean picoplankton includes prokaryotes (both bacteria and archaea) and tiny unicellular eukaryotes (hereafter picoeukaryotes), which feature fundamental differences in terms of cellular structure, feeding habits, metabolic diversity, growth rates and behaviour $(10,11)$. Even though marine picoeukaryotes and prokaryotes are usually investigated separately, they are intimately connected through biogeochemical

97 and food web networks (12-14). Overall, given the large effects picoeukaryotes can

98 have on the populations of prokaryotes (and vice versa), it is highly relevant to determine whether or not their communities are structured by the action of similar ecological processes.

102 four processes: selection, dispersal, ecological drift and speciation (15-17). Selection

103 involves deterministic reproductive differences among individuals from different or the same species as a response to biotic or abiotic conditions. Selection can act in two opposite directions, it can constrain (homogeneous selection) or promote

106 (heterogeneous selection) the divergence of communities (18). Dispersal, the movement 107 of organisms across space, affects microbial assemblages by incorporating individuals 
originating from the regional species pool. Dispersal rates can be high (homogenising dispersal), moderate, or low [dispersal limitation] (18). Dispersal limitation occurs when species are absent from suitable habitats because potential colonizers are too far away (19). The importance of dispersal limitation increases as geographic scale increases (20). Ecological drift (hereafter drift) in a local community refers to random changes in species' relative abundances derived from the stochastic processes of birth, death and offspring generation $(15,17)$. The action of drift in a metacommunity, that is, local communities that are connected via dispersal of multiple species (21), may lead to neutral dynamics (20), where random dispersal is the main mechanism of community assembly. In this neutral scenario, if dispersal is not limited, local communities will tend to resemble random subsamples of the metacommunity (20). Finally, speciation is the emergence of new species by evolution $(15,17)$, and it will not be considered hereafter as it is expected to have a small impact in the turnover of communities that are connected via dispersal (22).

The interplay of selection, dispersal and drift may generate different microbial assemblages that could feature diverse metabolisms and ecologies (16). The action of selection (in moderate to high strength) together with moderate rates of dispersal may generate a deterministic coupling between specific environmental conditions and combinations of species, a spatial pattern known as species sorting (23). In contrast, high or low levels of dispersal may produce the opposite effect, that is, a decoupling between abiotic environmental conditions (i.e. selection) and species assemblages. Particularly, high dispersal rates may maintain populations in habitats to which they are maladapted (21). Inversely, low dispersal rates may preclude species from reaching suitable habitats, leading to species assemblages that become more different as the geographic distance between them increases (distance decay). Still, both selection and 
133 dispersal limitation can generate distance decay (24). Drift is expected to cause

134 important random effects in local community composition in cases where selection is

135 weak and populations are small $(16,25)$.

136 Whereas global-ocean connectivity patterns reveal the importance of dispersal in

137 structuring communities in the upper ocean (26), our understanding of the relative role

138 of selection, dispersal and drift in structuring the global-ocean microbiome is still poor

$139(24,27,28)$. Multiple studies in diverse environments indicate that selection has a major

140 role in structuring prokaryotic communities $(23,24)$, although there is also evidence

141 pointing to drift as having a structuring role $(29,30)$. Here, we examine the mechanisms

142 shaping the sunlit global-ocean microbiome by addressing the following questions:

143 What are the relative roles of selection, dispersal and drift in shaping assemblages of

144 prokaryotes and picoeukaryotes? What environmental variables exert selection? What

145 spatial patterns emerge from the action of these three processes? We hypothesize that

146 the major organismal differences between picoeukaryotes and prokaryotes (11) should

147 result in a different relative importance of selection, dispersal and drift in structuring

148 their communities. Specifically, we hypothesize that the lower capacity for dormancy of

149 picoeukaryotes $(11,31)$ should result in a larger dispersal limitation when compared to

150 prokaryotes, given that dormancy may allow prokaryotes transiting through large

151 geographic areas that are unsuitable for growth. Furthermore, given that some

152 prokaryotes may engage into metabolic cooperation (32), an uncommon behaviour in

153 picoeukaryotes, we expect selection to generate more co-occurrences among

154 prokaryotes than among picoeukaryotes. 
RESULTS

Processes shaping the surface global-ocean microbiome

We analysed the prokaryotes and picoeukaryotes composing this microbiome in

161 Based on these genes, we delineated Operational Taxonomic Units (OTUs) as species

162 proxies (see Methods). We applied an innovative methodology based on phylogenetic and species turnover (22) that allowed us to quantify the relative importance of selection, dispersal and drift (See Methods). prokaryotic communities, while dispersal limitation was the dominant force structuring

167 picoeukaryotic communities (Fig. 1). Selection explained $\sim 34 \%$ of the turnover of prokaryotic communities, and $\sim 17 \%$ of that in picoeukaryotes (Fig. 1). Heterogeneous selection had a relatively higher importance in structuring picoeukaryotes as compared to prokaryotes ( $\sim 16 \%$ vs. $\sim 9 \%$, respectively), while homogeneous selection was more important in structuring prokaryotic $(\sim 24 \%)$ than picoeukaryotic $(\sim 1 \%)$ communities

172 (Fig. 1). Dispersal limitation was by far the most important process structuring

173 picoeukaryotic communities $(\sim 76 \%)$, while this process had a lower importance in

174 prokaryotes $(\sim 35 \%)$ [Fig. 1]. Drift explained a larger fraction of community turnover in 175 prokaryotes $(31 \%)$ than in picoeukaryotes ( 6\%) [Fig. 1]. Homogenizing dispersal had 176 a very limited role in the structuring of the global ocean microbiome $(<1 \%$ for both 177 picoeukaryotes and prokaryotes).

178 Given that communities of prokaryotes and picoeukaryotes were predominantly 179 structured by different processes, we expected both groups to present contrasting B180 diversity patterns. Accordingly, we found only moderate correlations between Bray- 
181 Curtis and generalized UniFrac (gUniFrac) B-diversity indices between picoeukaryotes

182 and prokaryotes (Bray Curtis: $\rho=0.58$, gUniFrac: $\rho=0.61, \mathrm{p}=0.01$, Mantel tests; Fig.

183 S2). Rare species tend to occupy less sites than more abundant species (34), therefore

184 communities featuring different proportions of abundant and rare species may display

185 different spatial turnover. We found that picoeukaryotes had proportionally more

186 regionally rare species (here defined as those with mean abundances below $0.001 \%$ )

187 than prokaryotes (71\% vs. $48 \%$ of the species respectively) [Table S1, Fig. S3]. This is

188 consistent with the observation that picoeukaryotes had more restricted species

189 distributions (i.e., occurring in $<20 \%$ of the communities) than prokaryotes [95\% vs.

$19088 \%$ of the species respectively] (Table S2; Fig. S3).

\section{Selection acting on the microbiome}

We investigated the abiotic agents exerting selection in the ocean microbiome

by analysing the compositional differences between communities (ß-diversity) together

with a set of environmental variables considered in the Meta-119 dataset (See

Supplementary Information). We used different ß-diversity indices (Bray-Curtis,

TINA $_{\mathrm{w}}$, PINA $_{\mathrm{w}}$, gUniFrac; See Methods), as each captures distinct features of

community differentiation. Water temperature was the most important driver of

200 selection on prokaryotes (Fig. 2). Furthermore, water temperature appeared to affect

204 lower proportions (Fig. 2). In contrast, temperature had limited effects on

205 picoeukaryotic species associations (Fig. 2). Our results were further confirmed by 
independent data from the global surface-ocean collected during the TARA Oceans expedition (36), as both the Malaspina and TARA Oceans datasets displayed stronger

210 feature co-occurring prokaryotic species, with this pattern disappearing as the temperature difference between stations increases.

213 (Supplementary Information, Fig. S4), showed that fluorescence (a proxy for

214 Chlorophyll $a$ concentration) explained $31 \%$ of PINA $_{\mathrm{w}}$-based prokaryotic community

215 variance (PERMANOVA $R^{2}$ ), while it was not significant for picoeukaryotes (Fig. S5;

216 PINA $_{\mathrm{w}}$ is a phylogeny-based $\beta$-diversity index, See Methods). The remaining combinations of environmental variables and $\beta$-diversity metrics explained a minor

218 fraction of community variance, suggesting that abiotic selection, at the whole microbiome level, operates via a very limited set of environmental variables, largely temperature. particularly in prokaryotes suggests that prokaryotes and picoeukaryotes may show different patterns of species co-occurrences and co-exclusions in association networks

224 (37). We found that prokaryotes were more associated between themselves than

225 picoeukaryotes in networks considering co-occurrences and co-exclusions as well as in 226 networks including only co-occurrences (Fig. S6). Specifically, in networks including

227 both co-occurrences and co-exclusions, prokaryotes featured $\sim 33 \%$ of connected species (i.e. prokaryotic species with at least one association to another prokaryotic species) and an average number of associations per species (i.e. average degree) of $\sim 14$,

230 while picoeukaryotes displayed $\sim 17 \%$ of connected species and an average degree of $\sim 8$ 
231 (Table S3; Fig. S6). Networks including co-occurrences only displayed similar patterns

232 (Table S3; Fig. S6). The prokaryotic network was more modular, containing a higher

233 number of highly-connected clusters of species than the picoeukaryotic network (Table 234 S3).

The previous results were supported by analyses using the Maximal Information

236 Coefficient [MIC], which quantifies a wide array of functional and non-functional,

237 linear and non-linear, associations (38). MIC results indicated that prokaryotes had

238 more associations between themselves than picoeukaryotes (Table S4), a pattern that

239 was also observed in other data from the upper global ocean collected during the TARA

240 Ocean expedition (Table S5). Most associations detected by MIC were non-linear

241 [defining nonlinearity as MIC- $\rho^{2}>0.2$; (38)] [Table S4 \& S5], pointing to complex

242 associations that may be missed by classic correlation analyses, which evaluate linear

243 relationships.

\section{Selection acting on species}

246 The potential effects of selection on single species was evaluated by determining

247 their individual correlations with multiple environmental variables using the MIC (38).

248 In these analyses, temperature was the variable with the highest number of associated

249 prokaryotic species (1.7\%) when considering a MIC threshold $\geq 0.4$, representing $\sim 17 \%$

250 of the total estimated species abundance, while picoeukaryotic species displayed a

251 considerable smaller proportion ( $\sim 0.3 \%$ of the species representing $\sim 5 \%$ of the

252 estimated species abundance) [Fig. S7]. Picoeukaryotic and prokaryotic species also

253 had associations with oxygen, conductivity and salinity (Fig. S7), which co-vary with

254 temperature. The remaining environmental variables tested had limited associations

255 with individual species (Fig. S7), thus agreeing with our previous results suggesting that 
selection on the surface ocean microbiome operates via a limited set of environmental

260 prokaryotes than on picoeukaryotes in the surface global ocean. Our results were further

261 validated by analyses using data from the global TARA Oceans cruise, which indicated that prokaryotic species were associated predominantly with temperature and oxygen,

\section{Dispersal limitation}

Our quantifications indicated that dispersal limitation was almost twice as

Environmental conditions between pairs of adjacent stations over the trajectory of the cruise, typically separated by $250-500 \mathrm{~km}$, are generally comparable (i.e. selective differences between stations tend to be low in the tropical and subtropical ocean). Therefore, compositional differences between neighbouring communities could manifest dispersal limitation. Following these premises, we analysed the change in

274 picoeukaryotic and prokaryotic community composition along the trajectory of the

275 cruise by comparing each community to the one sampled immediately before in a

276 sequential manner (i.e. sequential $\beta$-diversity) [Fig. 4]. Both picoeukaryotic and

277 prokaryotic communities displayed variable amounts of sequential $\beta$-diversity (Fig. 4,

278 Panels A and B), although picoeukaryotes featured, on average, a higher sequential $\beta$ - 
$\beta$-diversity, which was significantly higher for picoeukaryotes than for prokaryotes

281 (Fig. S8).

282

Simultaneous action of selection and dispersal limitation

When geographic distance is correlated with environmental variation, spatial

community variance may be the manifestation of both selection and dispersal limitation.

We analysed community variance associated to different marine biogeographic

provinces, as defined by Longhurst (39) based on nutrient concentration, structure of the water column, wind regimes, satellite-derived primary production and composition of abundant phytoplankton species. After removing the effects of the most important environmental variables that were correlated with these geographic regions and that provinces still accounted for $\sim 20 \%$ of picoeukaryotic community variance when using Bray-Curtis and gUniFrac $\beta$-diversity indices (PERMANOVA $R^{2}$ ) [Fig. 2]. Likewise, tested $\beta$-diversity indices (PERMANOVA $R^{2}$ ) [Fig. 2]. The variability in community composition associated to these provinces most likely represent dispersal limitation, even though abiotic or biotic selection exerted by unmeasured variables cannot be ruled out. ß-diversity in picoeukaryotes and prokaryotes displayed positive correlations with distance (i.e. distance decay) predominantly within 1,000 km (Fig. 5), although correlations were weaker in prokaryotes than in picoeukaryotes, being consistent with a higher dispersal limitation in picoeukaryotes than in prokaryotes (Fig. 1). 
total of 14 communities where sequential $\beta$-diversity displayed abrupt changes, with 11

306 of them coinciding for both picoeukaryotes and prokaryotes [Fig. 4, Panels A \& B].

307 The Local Contribution to Beta Diversity (LCBD) index (40) (Fig. 4, Panel D)

308 indicated that $\sim 22 \%$ of both picoeukaryotic and prokaryotic communities (26 stations

309 each, totaling 36 stations) contributed the most to the $\beta$-diversity, with 16 communities

310 coinciding for both prokaryotes and picoeukaryotes $(p<0.05$; Fig. 4, Panel D, Table

311 S7). In addition, 8 of the 36 stations featuring a significant LCBD were also identified

312 as zones of abrupt community change in sequential Bray Curtis analyses (Table S7).

313 These zones featuring abrupt community change in both prokaryotes and

314 picoeukaryotes point to selection or dispersal acting simultaneously and strongly upon

315 both life's domains. 


\section{DISCUSSION}

319 Our assessment of the tropical and sub-tropical sunlit fraction of the global-

320 ocean microbiome during the Malaspina 2010 Circumnavigation Expedition indicated

321 that dispersal limitation is the dominant process structuring picoeukaryotic

322 communities, while selection, dispersal and drift have a balanced importance in

323 structuring prokaryotic communities. These results summarise the general action of

324 ecological processes at the microbiome level and cannot be extended to every single

325 species or group; for example, some picoeukaryotes display cosmopolitan distributions

326 (41). Our results also reflect the action of ecological processes in the tropical and

327 subtropical sunlit ocean. Therefore, considering other zones, such as the polar oceans,

328 could modify the relative importance of these processes. To determine the action of

329 selection, we used the principle indicating that phylogenetically closely related taxa

330 tend to be ecologically similar (and vice versa). This principle was supported by data

331 from prokaryotes and unicellular eukaryotes (42-45). Yet, there can be exceptions (46),

332 and failure to detect selection could inflate the estimates of dispersal limitation in our

333 methodology. We consider that dispersal limitation in picoeukaryotes was not inflated

334 in our analyses, as picoeukaryotes generally displayed more limited spatial distributions

335 than prokaryotes and a higher sequential $\beta$-diversity. Drift was pragmatically analysed

336 as the compositional variation between communities that does not differ from random

337 community assembly, thus representing neutral metacommunity dynamics $(20,22,44)$.

338 Hence, our quantifications of drift do not reflect the impact of random demography in

339 single communities. Lastly, our estimates of the importance of ecological mechanisms

340 consider taxa with high or moderate abundances, which typically carry biogeographic

341 information (47), thus, our results do not reflect the processes shaping the rare biosphere

342 (48). 

communities $(23,24)$, explained a higher proportion of community turnover in

345 prokaryotes $(\sim 34 \%$ of the turnover $)$ than in picoeukaryotes $(\sim 17 \%$ of the turnover $)$. This

346 modest role of selection in structuring the tropical and subtropical sunlit-ocean

347 microbiome is consistent with the weak environmental gradients characterizing this

348 habitat. In other habitats featuring a high selective pressure, such as Antarctic

349 waterbodies that display a strong salinity gradient, the role of selection in structuring

350 bacteria has been reported to be much higher, accounting for up to $\sim 70 \%$ of the

351 community turnover (49). The measured relative importance of selection is also a

352 consequence of the global scale of our survey. For example, in comparatively small

353 marine areas, where dispersal limitation is expected to be low (19), the relative

354 importance of selection could increase. In surface waters of the East China Sea it was

355 found that selection was $\sim 40 \%$ more important than dispersal limitation in structuring

356 bacterial communities (50), while in our study, selection and dispersal limitation had a

357 similar importance in structuring prokaryotes. The same study (50) also found that

358 selection was $\sim 40$ times more important than dispersal limitation in structuring

359 communities of microbial eukaryotes. In contrast, our global assessment yields dispersal

360 limitation to be $\sim 5$ times larger than selection in structuring picoeukaryotic

361 communities.

Heterogeneous selection was more important in structuring picoeukaryotic $(\sim 16 \%)$ than prokaryotic communities $(\sim 9 \%)$, while homogeneous selection was more

364 important in prokaryotic $(\sim 24 \%)$ than in picoeukaryotic communities $(\sim 1 \%)$.

365 Homogeneous environmental conditions should lead to homogeneous selection,

366 constraining community divergence, while heterogeneous environmental conditions

367 should promote community divergence (18). Our results suggest that prokaryotes and 
368 picoeukaryotes in the same marine habitats respond differently to the same

369 environmental conditions. Thus, selection would be preventing community divergence

370 in prokaryotes while promoting it in picoeukaryotes. The fundamental cellular

371 differences between prokaryotes and picoeukaryotes $(10,11)$ may determine such

372 different responses to the same environmental heterogeneity. For example, comparable

373 environmental heterogeneity could select for a few species featuring wide

374 environmental tolerance or several species, which are adapted to narrow environmental

375 conditions.

376 Previous studies indicate that temperature is one of the most important variables

377 exerting selection on ocean prokaryotes (51-59). Earlier work (51) reported strong

378 correlations between prokaryotic ocean-microbiome composition and temperature, and

379 weak correlations with nutrients, consistent with our results. Less is known about the

380 effects of temperature on the community structuring of ocean picoeukaryotes, which

381 according to our results are minor. Yet, it is known that temperature affects the

382 distributions of MAST-4, a lineage of ubiquitous marine heterotrophic flagellates (41),

383 suggesting that the effects of temperature on small eukaryotes could be group specific.

384 Interestingly, our results suggest that selection, operating via temperature, affects

385 prokaryotic taxa co-occurrences, having limited effects on picoeukaryotic co-

386 occurences. In prokaryotes, the $\beta$-diversity associated to temperature explained by

387 TINA $_{\mathrm{w}}(\sim 50 \%)$ was substantially higher than Bray Curtis $(\sim 15 \%)$, reflecting the

388 importance of considering co-occurrences, as in $\mathrm{TINA}_{\mathrm{w}}$, to understand community

389 structure. These results suggest that temperature-driven selection determines the species

390 that can grow in different locations, yet in each site, species relative abundances and

391 presence-absence may vary due to local stochasticity $(60,61)$. 

expected to be distributed more thoroughly than the less abundant picoeukaryotes (34), which is consistent with our findings. Furthermore, the absence of taxa from suitable habitats that are separated by large distances is expected to be more pronounced in picoeukaryotes than in prokaryotes. Still, our analyses compare actual species distributions against those that would be expected by chance when considering species

401 abundances, suggesting that species-abundance is not the main reason of dispersal

402 limitation in picoeukaryotes. coccolitophorids $(74,75)$, which may increase dispersal rates. Cyst formation has not been reported yet for picoeukaryotes (11) and this may partially explain their limited dispersal. Regarding prokaryotes, other studies considering large geographic scales indicated that dispersal limitation has a small influence in the structure of marine

411 communities $(51,52,76)$, which is coherent with our results. Dormancy in prokaryotes

412 seems to be more common than in picoeukaryotes $(11,31)$, and this may allow the

413 former to disperse more thoroughly by reducing their metabolisms when moving

414 through unfavorable habitats (77). In sum, our quantifications of dispersal limitation

415 agree, in general terms, with known trends in both picoeukaryotes and prokaryotes. 
417 communities is also a matter of debate $(23,78)$. Our results indicate that drift has a

418 modest role in structuring the sunlit-ocean microbiome, being higher in prokaryotic

$419(\sim 31 \%$ of the turnover) than in picoeukaryotic communities ( $\sim 6 \%$ of the turnover).

420 Another study also found a higher importance of drift in determining the community

421 structure of bacteria when compared with phytoplankton populating freshwater and

422 brackish habitats (79). In contrast, drift was the prevalent community-structuring

423 mechanism in unicellular eukaryotes populating lakes in a relatively small geographic

424 area that features a strong salinity gradient, having a low importance for the structuring

425 of prokaryotic communities (49). Likely, the relative importance of drift in structuring

426 prokaryotes or unicellular eukaryotes is dependent on the selective strength of specific

427 habitats, the occurrence of adaptive processes (49) or barriers to dispersal.

428 When geographic distance is correlated with environmental variation, a decrease

429 in community similarity with distance (distance decay) can be the manifestation of both

430 selection and/or dispersal limitation (24). Distance decay has been evidenced in diverse

431 studies focusing on the surface and deep ocean microbiome $(52,80,81)$. Yet, different

432 to most previous studies, we have quantified the role of selection and dispersal

433 limitation in structuring the surface ocean microbiome, and we can use this information

434 to interpret the measured distance decay. As our quantifications indicated a strong

435 dispersal limitation in picoeukaryotes, it is likely that this process explains the measured

436 distance decay. In contrast, the distance decay observed in prokaryotes could be the

437 outcome of both selection and dispersal limitation, as both presented comparable

438 structuring roles. The amount of community variance in prokaryotes and picoeukaryotes

439 associated to provincialism (i.e. Longhurst oceanic regions) likely reflects dispersal

440 limitation, since the effects of important environmental variables were removed during 
441 the analyses. Interestingly, another study investigating surface marine bacteria along

$442 \sim 12,000 \mathrm{~km}$ in the Atlantic Ocean found that provincialism explained an amount of

443 community variance comparable to our results (52). Furthermore, a study of the

444 eukaryotic microbiome in the sunlit global-ocean indicated that provincialism

445 (considered in terms of ocean basins) was one of the most important variables

446 explaining community structure (67). In the light of our findings, we consider that

447 results from the previous study (67) manifest, to a large extent, dispersal limitation.

448 In the surface ocean, drastic changes in species composition across space may

449 point to strong changes in abiotic selection, as expected to occur across oceanographic

450 fronts $(82,83)$, or high immigration from the surface or deeper water layers. We

451 identified 14 stations featuring abrupt changes in prokaryotic or picoeukaryotic

452 community composition as well as 36 stations with a "unique" species composition

453 according to the Local Contribution to Beta Diversity analysis (40). Several of these

454 stations coincided for both picoeukaryotes and prokaryotes. Some of these areas

455 correspond to nutrient-rich coastal zones (the South African Atlantic coast and the

456 South Australia Bight) or potential upwelling zones, such as the Equatorial Pacific and

457 Atlantic as well as the Costa Rica Dome. This agrees with a scenario including strong

458 selective changes or immigration from deep water layers into the surface, which affects

459 both prokaryotes and picoeukaryotes.

$460 \quad$ Altogether, our results represent a significant contribution towards

461 understanding the structure of the sunlit-ocean microbiome by connecting patterns to

462 underlying ecological processes (24). Our findings indicate that comprehending the

463 idiosyncrasies of the main components of microbiomes is needed in order to attain a

464 holistic understanding of their structures and ecologies. In particular, our results suggest

465 that the structure of surface-ocean prokaryotic communities could be more susceptible 
466 to global warming than that of picoeukaryotic communities. Prokaryotes represent an

467 important fraction of the total microbial biomass in the ocean $(84,85)$, and they have

468 fundamental roles for ecosystem function $(1,2)$. Therefore, understanding the specific

469 effects of temperature in their distributions and community metabolism (27) represents

470 an important challenge, which our results contribute to address. 


\section{METHODS}

472

\section{Sample collection}

Surface waters ( $3 \mathrm{~m}$ depth) from a total of 120 globally-distributed stations

475 located in the tropical and sub-tropical global ocean (Fig. S1) across $\sim 50,000 \mathrm{~km}$, were sampled from December 2010 to July 2011 as a part of the Malaspina-2010 expedition

$477(33,86)$. Water samples were obtained with a 20 L Niskin bottle deployed simultaneously to a CTD profiler that included sensors for conductivity, temperature, oxygen, fluorescence and turbidity. About $12 \mathrm{~L}$ of seawater were sequentially filtered 480 through a $20 \mu \mathrm{m}$ nylon mesh, followed by a $3 \mu \mathrm{m}$ and $0.2 \mu \mathrm{m}$ polycarbonate filters of $48147 \mathrm{~mm}$ diameter (Isopore, Millipore). Only the smallest size-fraction [0.2 -3 $\mu \mathrm{m}$, here called "picoplankton"; see (5)] was used in downstream analyses. Samples for inorganic nutrients $\left(\mathrm{NO}_{3}^{-}, \mathrm{NO}_{2}^{-}, \mathrm{PO}_{4}{ }^{3-}, \mathrm{SiO}_{2}\right)$ were collected from the Niskin bottles and measured spectrophotometrically using an Alliance Evolution II autoanalyzer (87). Chlorophyll measurements were obtained from Estrada et al. (86). In specific samples nutrient concentrations were estimated using the World Ocean Database (88-91) due to issues stations, two contextual datasets were generated: Meta-119, including 119 stations (Fig.

S1), 5 environmental parameters and 5 spatial features and Meta-57 (Fig. S4), including further details.

DNA extraction, 18S- \& 16S-rRNA amplicon sequencing and bioinformatic analyses 
hypervariable V4 region of the $18 \mathrm{~S}(\sim 380 \mathrm{bp})$ was amplified with the primers

TAReukFWD1 and TAReukREV3 (93), while the hypervariable V4-V5 ( 400bp)

500 Illumina MiSeq platform (2x250bp) at the Research and Testing Laboratory facility

501 (Lubbock, TX, USA; http://www.researchandtesting.com/). de-replicated using USEARCH (99). Operational Taxonomic Units (OTUs) were

506 delineated at 99\% similarity using UPARSE V8.1.1756 (100). To obtain OTU

507 abundances, reads were mapped back to OTUs at 99\% similarity. Chimera check and

508 removal was performed both de novo and using the SILVA reference database (101).

509 After our stringent quality control (see Supplementary Information), a total of 42,505

510 picoeukaryotic and 10,158 prokaryotic OTUs were obtained. Taxonomic assignment of

511 picoeukaryotic and prokaryotic OTUs was generated by BLASTing (102) representative sequences against different reference databases. Metazoan, Charophyta, nucleomorphs,

513 Chloroplast and mitochondrial OTUs were removed from the OTU tables. See more

514 details in Supplementary Information and Table S8. Sequences are publicly available at

515 the European Nucleotide Archive (http://www.ebi.ac.uk/ena; accession numbers

516 PRJEB23913 [18S] \& PRJEB25224 [16S]).

518 Oceans expedition (36). We selected data from surface communities only, including 41

519 samples (40 stations) for pico-nano eukaryotes $(0.22-3 \mu \mathrm{m}$ [1 sample] and 0.8-5 $\mu \mathrm{m}$ [40 
521 [picoplankton, 0.22-3 $\mu \mathrm{m}$ (45 samples) and 0.22-1.6 $\mu \mathrm{m}$ (18 samples); 16S rDNA,

522 miTags] (51).

523

524 General analyses and phylogenetic inferences

525 Both picoeukaryotic and prokaryotic datasets were sub-sampled to 4,060 reads

526 per sample using rrarefy in Vegan (103), resulting in sub-sampled tables containing

52718,881 picoeukaryotic and 7,025 prokaryotic OTUs. All OTUs with mean relative

528 abundances above $0.1 \%$ and below $0.001 \%$ were defined as regionally abundant or rare

529 respectively (104). Phylogenetic trees were constructed for both the 16S and 18S

530 datasets using OTU-representative sequences. Reads were aligned against an aligned

531 SILVA template using mothur (105). Afterwards, poorly aligned regions or sequences

532 were removed using Trimal (106). A phylogenetic tree was inferred using FastTree

533 v2.1.9 (107). Most analyses were performed in the R statistical environment (108) using

534 adespatial (109), APE (110), ggplot2 (111), gUniFrac (112), Maps (113), Mapplots

535 (114), Picante (115) and Vegan (103). See further details in Supplementary

536 Information.

537

\section{Quantification of selection, dispersal and drift}

539 Selection, dispersal and drift were quantified using the approach proposed by

540 Stegen et al. (22). This methodology consists of two main steps: the first uses

541 phylogenetic turnover to infer the action of selection and the second uses OTU turnover

542 to infer the action of dispersal and drift. Phylogenetic turnover was measured by

543 calculating the abundance-weighted $\beta$-mean nearest taxon distance ( $\beta$ MNTD), which

544 quantifies the mean phylogenetic distances between the evolutionary closest OTUs in

545 two communities. $\beta$ MNTD values can be larger, smaller or equal to the values expected 
546 when selection is not affecting community turnover (that is, expected under a random

547 distribution [null model]). $\beta$ MNTD values higher than expected by chance indicate that

548 communities are under heterogeneous selection (18). In contrast, $\beta$ MNTD values which

549 are lower than expected by chance indicate that communities are experiencing

550 homogeneous selection. Null models were constructed using 999 randomizations as in

551 Stegen et al. (22). Differences between the observed $\beta$ MNTD and the mean of the null

552 distribution are denoted as $\beta$-Nearest Taxon Index ( $\beta$ NTI), with $|\beta N T I|>2$ being

553 considered as significant departures from random phylogenetic turnover, pointing to the

554 action of selection.

555 The second step of this method calculates whether the observed $\beta$-diversity,

556 based on OTU turnover, could be generated by drift (i.e. chance) or other processes. For

557 this, we calculated the Raup-Crick metric (116) using Bray-Curtis dissimilarities

558 [hereafter $\mathrm{RC}_{\text {bray }}$, following Stegen et al. (22). $\mathrm{RC}_{\text {bray }}$ compares the measured $\beta$ -

559 diversity against the $\beta$-diversity that would be obtained if drift was driving community

560 turnover (that is, under random community assembly). The randomization was run

5619,999 times and only OTUs with $>1,000$ reads over the entire dataset were considered in

562 order to prevent any bias due to potential under sampling. $\mathrm{RC}_{\text {bray }}$ values between -0.95

563 and +0.95 point to a community assembly governed by drift. On the contrary, $\mathrm{RC}_{\text {bray }}$

564 values $>+0.95$ or $<-0.95$ indicate that community turnover is driven by dispersal

565 limitation or homogenizing dispersal respectively (116). The previous framework was

566 applied as following: First, we determined the proportion of community pairwise

567 comparisons displaying a $|\beta \mathrm{NTI}|>2$, which points to the action of selection.

568 Subsequently, for the pairwise comparisons that did not indicate the action of selection,

569 we calculated the proportion of total comparisons that could be assigned to dispersal 
570 limitation, homogenizing dispersal or drift according to their $\mathrm{RC}_{\text {bray }}$ values. See further

571 details in Supplementary Information.

572

\section{$573 \quad$ Estimation of interaction-adjusted indices}

Taxa INteraction-Adjusted (TINA) and Phylogenetic INteraction Adjusted

575 (PINA) indices were estimated following Schmidt et al. (35) TINA is based on taxa co-

576 occurrences while PINA considers phylogenetic similarities (35). In particular, TINA

577 quantifies $\beta$-diversity as the average interaction strength between all taxa in different

578 samples. Thus, communities which are identical or include taxa which are perfectly

579 associated will give TINA values of 1 . On the other hand, TINA values will approach

5800.5 in communities sharing no taxa or having neutral associations, and approach 0 if

581 taxa display high avoidance. Dissimilarity matrices were generated as 1-TINA. Full

582 picoeukaryotic and prokaryotic subsampled OTU tables were used to calculate the

583 abundance-weighted TINA ${ }_{w}$ and PINA $_{w}$.

584

585

Associations between taxa and environmental parameters

586

We analysed whether OTUs had differential associations with environmental

587 parameters as well as between themselves using different algorithms. Firstly, we used

588 the Maximum Information Coefficient (MIC) which captures diverse relationships

589 (including non-linear and non-functional) between two pairs of variables (38). The

590 Malaspina dataset consisted of 119 stations and 17 environmental parameters (See

591 Supplementary Information for extra details). In the TARA Oceans dataset, prokaryotes

592 were analysed across 63 surface stations (including 8 environmental parameters), while

593 microbial eukaryotes were analysed across 40 surface stations (including 6

594 environmental parameters) [see Supplementary Information]. In both datasets, MIC 
595 analyses were run using $\mathrm{CV}=0.5, \mathrm{~B}=0.6$, and statistically significant relationships with

596 MIC $\geq 0.4$ (Malaspina) or MIC $\geq 0.5$ (TARA) were considered; significance was

597 assessed using precomputed p-values (38). Non-linear associations were defined as

598 MIC- $\rho^{2}>0.2$ (38). Secondly, we constructed association networks with the Malaspina

599 dataset considering OTUs with $>100$ reads using SparCC (117) as implemented in

600 FastSpar (118). To determine correlations, FastSpar was run with 1,000 iterations,

601 including 1,000 bootstraps to infer p-values. We used OTU associations with absolute

602 correlation scores $>0.3$ and $\mathrm{p}<0.01$. Networks were visualized with Cytoscape (119) and

603 their properties determined using igraph (120).

604 


\section{ACKNOWLEDGEMENTS}

606 We thank all scientists and crews from the Malaspina-2010 expedition. RL was

607 supported by a Ramón y Cajal fellowship (RYC-2013-12554, MINECO, Spain). IMD

608 was supported by an ITN-SINGEK fellowship (ESR2-EU-H2020-MSCA-ITN-2015,

609 Grant Agreement 675752) and CRG by a Juan de la Cierva (IJCI-2015-23505,

610 MINECO, Spain) fellowship. This work was supported by the projects Malaspina-2010

611 Expedition (CSD2008-00077, MINECO, Spain), INTERACTOMICS (CTM2015-

612 69936-P, MINECO, Spain), REMEI (CTM2015-70340-P, MINECO, Spain) and

613 MicroEcoSystems (240904, RCN, Norway). Bioinformatics analyses were performed at

614 the MARBITS platform of the Institut de Ciències del Mar (ICM;

615 http://marbits.icm.csic.es) as well as in MareNostrum (Barcelona Supercomputing

616 Center) via grants obtained from the Spanish Network of Supercomputing (RES) to RL.

617 
618

619

620

621

622

623

624

625

626

627

628

629

630

631

632

633

634

635

636

637

638

639

640

641

642

643

644

645

646

647

648

649

650

651

652

653

654

\section{REFERENCES}

1. Falkowski P (2012) The power of plankton. Nature 483(7387):S17-20.

2. Falkowski PG, Fenchel T, \& Delong EF (2008) The microbial engines that drive Earth's biogeochemical cycles. Science 320(5879):1034-1039.

3. Pedrós-Alió C, Acinas SG, Logares R, \& Massana R (2018) Marine microbial diversity as seen by high throughput sequencing. Microbial Ecology of the Oceans, eds Gasol JM \& Kirchman DL (Wiley-Blackwell, Hoboken, New Jersey), p 592.

4. Worden AZ, et al. (2015) Rethinking the marine carbon cycle: factoring in the multifarious lifestyles of microbes. Science 347(6223):1257594.

5. Massana R (2011) Eukaryotic picoplankton in surface oceans. Annual review of microbiology 65:91-110.

6. Jardillier L, Zubkov MV, Pearman J, \& Scanlan DJ (2010) Significant $\mathrm{CO}_{2}$ fixation by small prymnesiophytes in the subtropical and tropical northeast Atlantic Ocean. ISME J 4(9):1180-1192.

7. Li WKW (1994) Primary production of prochlorophytes, cyanobacteria, and eucaryotic ultraphytoplankton: Measurements from flow cytometric sorting. Limnology and Oceanography 39(1):169-175.

8. Field CB, Behrenfeld MJ, Randerson JT, \& Falkowski P (1998) Primary production of the biosphere: integrating terrestrial and oceanic components. Science 281(5374):237-240.

9. del Giorgio PA \& Duarte CM (2002) Respiration in the open ocean. Nature 420(6914):379-384.

10. Keeling PJ \& Del Campo J (2017) Marine Protists Are Not Just Big Bacteria. Curr Biol 27(11):R541-R549.

11. Massana R \& Logares R (2013) Eukaryotic versus prokaryotic marine picoplankton ecology. Environ Microbiol 15(5):1254-1261.

12. Massana R (2009) Picoeukaryotes. Encyclopedia of Microbiology, ed Schaechter M (Elsevier, Oxford), pp 674-688.

13. Seymour JR, Amin SA, Raina JB, \& Stocker R (2017) Zooming in on the phycosphere: the ecological interface for phytoplankton-bacteria relationships. Nat Microbiol 2:17065.

14. Jürgens K \& Massana R (2008) Protistan grazing on marine bacterioplankton (Wiley-Blackwell, Hoboken, New Jersey) 2nd Ed pp 383-441.

15. Vellend M (2010) Conceptual synthesis in community ecology. Q Rev Biol 85(2):183-206. 
16. Nemergut DR, et al. (2013) Patterns and processes of microbial community assembly. Microbiol Mol Biol Rev 77(3):342-356.

17. Vellend M (2016) The theory of ecological communities (Princeton University Press, Princeton) pp ix, 229 pages.

18. Zhou J \& Ning D (2017) Stochastic Community Assembly: Does It Matter in Microbial Ecology? Microbiol Mol Biol Rev 81(4):e00002-00017.

19. Heino J, et al. (2015) Metacommunity organisation, spatial extent and dispersal in aquatic systems: patterns, processes and prospects. Freshwater Biology 60(5):845-869.

20. Hubbell SP (2001) A unified neutral theory of biodiversity and biogeography (Princeton University Press, Princeton, NJ).

21. Holyoak M, Leibold MA, \& Holt RD (2005) Metacommunities: Spatial Dynamics and Ecological Communities (The University of Chicago Press, Chicago).

22. Stegen JC, et al. (2013) Quantifying community assembly processes and identifying features that impose them. ISME J 7(11):2069-2079.

23. Lindström ES \& Langenheder S (2012) Local and regional factors influencing bacterial community assembly. Environ Microbiol Rep 4:1-9.

24. Hanson CA, Fuhrman JA, Horner-Devine MC, \& Martiny JB (2012) Beyond biogeographic patterns: processes shaping the microbial landscape. Nature reviews. Microbiology 10(7):497-506.

25. Fisher CK \& Mehta $\mathrm{P}$ (2014) The transition between the niche and neutral regimes in ecology. Proc Natl Acad Sci U S A 111(36):13111-13116.

26. Villarino E, et al. (2018) Large-scale ocean connectivity and planktonic body size. Nature communications $9(1)$.

27. Graham EB, et al. (2016) Coupling Spatiotemporal Community Assembly Processes to Changes in Microbial Metabolism. Front Microbiol 7:1949.

28. Graham EB, et al. (2016) Microbes as Engines of Ecosystem Function: When Does Community Structure Enhance Predictions of Ecosystem Processes? Front Microbiol 7:214.

29. Ofiteru ID, et al. (2010) Combined niche and neutral effects in a microbial wastewater treatment community. Proc Natl Acad Sci U S A 107(35):1534515350.

30. Sloan WT, et al. (2006) Quantifying the roles of immigration and chance in shaping prokaryote community structure. Environ Microbiol 8(4):732-740.

31. Jones SE \& Lennon JT (2010) Dormancy contributes to the maintenance of microbial diversity. Proc Natl Acad Sci US A 107(13):5881-5886. 
706

707

708

709

710

711

712

713

714

715

716

717

718

719

720

721

722

723

32. Morris BE, Henneberger R, Huber H, \& Moissl-Eichinger C (2013) Microbial syntrophy: interaction for the common good. FEMS Microbiol Rev 37(3):384406.

33. Duarte CM (2015) Seafaring in the 21St Century: The Malaspina 2010 Circumnavigation Expedition. Limnology and Oceanography Bulletin 24(1):1114.

34. Gaston KJ, et al. (2000) Abundance-occupancy relationships. Journal of Applied Ecology 37(s1):39-59.

35. Schmidt TS, Matias Rodrigues JF, \& von Mering C (2017) A family of interaction-adjusted indices of community similarity. ISME J 11(3):791-807.

36. Karsenti E, et al. (2011) A holistic approach to marine eco-systems biology. PLoS biology 9(10):e1001177.

37. Faust K \& Raes J (2012) Microbial interactions: from networks to models. Nat Rev Microbiol 10(8):538-550.

38. Reshef DN, et al. (2011) Detecting novel associations in large data sets. Science 334(6062):1518-1524.

39. Longhurst AR (2007) Ecological Geography of the Sea (Academic Press).

40. Legendre P \& De Caceres M (2013) Beta diversity as the variance of community data: dissimilarity coefficients and partitioning. Ecol Lett 16(8):951-963.

41. Rodriguez-Martinez R, Rocap G, Salazar G, \& Massana R (2013) Biogeography of the uncultured marine picoeukaryote MAST-4: temperature-driven distribution patterns. ISME J 7(8):1531-1543.

42. Pyron RA, Costa GC, Patten MA, \& Burbrink FT (2015) Phylogenetic niche conservatism and the evolutionary basis of ecological speciation. Biol Rev Camb Philos Soc 90(4):1248-1262.

43. Losos JB (2008) Phylogenetic niche conservatism, phylogenetic signal and the relationship between phylogenetic relatedness and ecological similarity among species. Ecol Lett 11(10):995-1003.

44. Stegen JC, Lin X, Konopka AE, \& Fredrickson JK (2012) Stochastic and deterministic assembly processes in subsurface microbial communities. ISME J 6(9):1653-1664.

45. Violle C, Nemergut DR, Pu Z, \& Jiang L (2011) Phylogenetic limiting similarity and competitive exclusion. Ecol Lett 14(8):782-787.

46. Logares R, et al. (2007) Phenotypically different microalgal morphospecies with identical ribosomal DNA: A case of rapid adaptive evolution? Microbial Ecology 53(4):549-561. 
47. Ser-Giacomi E, et al. (2018) Ubiquitous abundance distribution of nondominant plankton across the global ocean. Nat Ecol Evol.

48. Pedrós-Alió C (2012) The rare bacterial biosphere. Ann Rev Mar Sci 4:449-466.

49. Logares R, et al. (2018) Contrasting prevalence of selection and drift in the community structuring of bacteria and microbial eukaryotes. Environ Microbiol.

50. $\mathrm{Wu} \mathrm{W}$, et al. (2018) Contrasting the relative importance of species sorting and dispersal limitation in shaping marine bacterial versus protist communities. ISME J 12(2):485-494.

51. Sunagawa S, et al. (2015) Structure and function of the global ocean microbiome. Science 348(6237):1261359.

52. Milici M, et al. (2016) Bacterioplankton Biogeography of the Atlantic Ocean: A Case Study of the Distance-Decay Relationship. Front Microbiol 7:590.

53. Delmont TO, et al. (2017) The global biogeography of amino acid variants within a single SAR11 population is governed by natural selection. bioRxiv.

54. Johnson ZI, et al. (2006) Niche partitioning among Prochlorococcus ecotypes along ocean-scale environmental gradients. Science 311(5768):1737-1740.

55. Delmont TO \& Eren AM (2018) Linking pangenomes and metagenomes: the Prochlorococcus metapangenome. PeerJ 6:e4320.

56. Simon M, Glöckner FO, \& Amann R (1999) Different community structure and temperature optima of heterotrophic picoplankton in various regions of the Southern Ocean. Aquat Microb Ecol 18(3):275-284.

57. Price PB \& Sowers T (2004) Temperature dependence of metabolic rates for microbial growth, maintenance, and survival. Proc Natl Acad Sci U S A 101(13):4631-4636.

58. Follows MJ, Dutkiewicz S, Grant S, \& Chisholm SW (2007) Emergent biogeography of microbial communities in a model ocean. Science 315(5820):1843-1846.

59. Zinser ER, et al. (2007) Influence of light and temperature on Prochlorococcus ecotype distributions in the Atlantic Ocean. Limnology and Oceanography 52(5):2205-2220.

60. Chase JM (2003) Community assembly: when should history matter? Oecologia 136(4):489-498.

61. Gonze D, Lahti L, Raes J, \& Faust K (2017) Multi-stability and the origin of microbial community types. ISME J 11(10):2159-2166.

62. Martiny JB, et al. (2006) Microbial biogeography: putting microorganisms on the map. Nat Rev Microbiol 4(2):102-112. 
63. Logares R (2006) Does the global microbiota consist of a few cosmopolitan species? Ecología Austral 16:85-90.

64. Foissner W (2006) Biogeography and Dispersal of Micro-organisms: A Review Emphasizing Protists. Acta Protozoologica 45:111-136.

65. Papke RT \& Ward DM (2004) The importance of physical isolation to microbial diversification. FEMS Microbiol. Ecol. 48(3):293-303.

66. Kirchman DL (2008) Microbial Ecology of the Oceans (John Wiley \& Sons, Hoboken, New Jersey).

67. de Vargas C, et al. (2015) Eukaryotic plankton diversity in the sunlit ocean. Science 348(6237):1261605.

68. Darling KF, Kucera M, \& Wade CM (2007) Global molecular phylogeography reveals persistent Arctic circumpolar isolation in a marine planktonic protist. Proc Natl Acad Sci U S A 104(12):5002-5007.

69. Casteleyn G, et al. (2010) Limits to gene flow in a cosmopolitan marine planktonic diatom. Proc Natl Acad Sci U S A 107(29):12952-12957.

70. Cermeño P, de Vargas C, Abrantes F, \& Falkowski PG (Phytoplankton biogeography and community stability in the ocean. PLoS One 5(4):e10037.

71. Cermeno P \& Falkowski PG (2009) Controls on diatom biogeography in the ocean. Science 325(5947):1539-1541.

72. Whittaker KA \& Rynearson TA (2017) Evidence for environmental and ecological selection in a microbe with no geographic limits to gene flow. Proc Natl Acad Sci U S A 114(10):2651-2656.

73. Bass D, Richards TA, Matthai L, Marsh V, \& Cavalier-Smith T (2007) DNA evidence for global dispersal and probable endemicity of protozoa. BMC Evol Biol 7(1):162.

74. Lewis J, Harris ASD, Jones KJ, \& Edmonds RL (1999) Long-term survival of marine planktonic diatoms and dinoflagellates in stored sediment samples. $J$ Plankton Res 21(2):343-354.

75. Billard C \& Inouye I (2004) What is new in coccolithophore biology? Coccolithophores: From Molecular Processes to Global Impact, eds Thierstein HR \& Young JR (Springer Berlin Heidelberg, Berlin, Heidelberg), pp 1-29.

76. Sintes E, De Corte D, Ouillon N, \& Herndl GJ (2015) Macroecological patterns of archaeal ammonia oxidizers in the Atlantic Ocean. Mol Ecol 24(19):49314942.

77. Locey KJ (2010) Synthesizing traditional biogeography with microbial ecology: the importance of dormancy. Journal of Biogeography 37(10):1835-1841. 
800 78. Louca S, et al. (2018) Function and functional redundancy in microbial systems. Nature Ecology \& Evolution 2:936-943.

802 79. Östman Ö, et al. (2010) Regional invariance among microbial communities.

803 Ecology letters 13(1):118-127.

804 80. Salazar G, et al. (2016) Global diversity and biogeography of deep-sea pelagic

805

806

81. Zinger L, Boetius A, \& Ramette A (2014) Bacterial taxa-area and distance-

807

808

82. Díez B, Massana R, Estrada M, \& Pedrós-Alió C (2004) Distribution of

809 eukaryotic picoplankton assemblages across hydrographic fronts in the Southern

810

811 Ocean, studied by denaturing gradient gel electrophoresis. Limnology and Oceanography 49(4):1022-1034.

83. Flaviani F, et al. (2018) Distinct oceanic microbiomes (from viruses to protists) found either side of the Antarctic Polar Front. Front Microbiol 9.

84. Bar-On YM, Phillips R, \& Milo R (2018) The biomass distribution on Earth.

85. Whitman WB, Coleman DC, \& Wiebe WJ (1998) Prokaryotes: the unseen majority. Proc Natl Acad Sci U S A 95(12):6578-6583.

86. Estrada M, et al. (2016) Phytoplankton across Tropical and Subtropical Regions

88. Boyer TP, et al. (2013) World Ocean Database 2013. in NOAA Atlas NESDIS

89. Catalá TS, et al. (2016) Drivers of fluorescent dissolved organic matter in the

90. Catalá TS, et al. (2016) Chromophoric signatures of microbial by-products in

91. Fernandez-Castro B, et al. (2015) Importance of salt fingering for new nitrogen and phylogenetic characterization of marine planktonic Archaea in the Santa Barbara Channel. Appl Environ Microbiol 63(1):50-56. water. Mol Ecol 19 Suppl 1:21-31. 
94. Parada AE, Needham DM, \& Fuhrman JA (2016) Every base matters: assessing small subunit rRNA primers for marine microbiomes with mock communities, time series and global field samples. Environ Microbiol 18(5):1403-1414.

95. Logares R (2017) Workflow for Analysing MiSeq Amplicons based on Uparse v1.5. (https://doi.org/10.5281/zenodo.259579).

96. Nikolenko SI, Korobeynikov AI, \& Alekseyev MA (2013) BayesHammer: Bayesian clustering for error correction in single-cell sequencing. $B M C$ Genomics 14 Suppl 1:S7.

97. Schirmer M, et al. (2015) Insight into biases and sequencing errors for amplicon sequencing with the Illumina MiSeq platform. Nucleic Acids Res 43(6):e37.

98. Zhang J, Kobert K, Flouri T, \& Stamatakis A (2014) PEAR: a fast and accurate Illumina Paired-End reAd mergeR. Bioinformatics 30(5):614-620.

99. Edgar RC (2010) Search and clustering orders of magnitude faster than BLAST. Bioinformatics 26(19):2460-2461.

100. Edgar RC (2013) UPARSE: highly accurate OTU sequences from microbial amplicon reads. Nat Methods 10(10):996-998.

101. Quast C, et al. (2013) The SILVA ribosomal RNA gene database project: improved data processing and web-based tools. Nucleic Acids Res 41(Database issue):D590-596.

102. Altschul SF, Gish W, Miller W, Myers EW, \& Lipman DJ (1990) Basic local alignment search tool. Journal of molecular biology 215(3):403-410.

103. Oksanen J, et al. (2008) vegan: Community Ecology Package. R package version $1.15-0$.

104. Logares R, et al. (2014) Patterns of rare and abundant marine microbial eukaryotes. Curr Biol 24(8):813-821.

105. Schloss PD, et al. (2009) Introducing mothur: open-source, platformindependent, community-supported software for describing and comparing microbial communities. Appl Environ Microbiol 75(23):7537-7541.

106. Capella-Gutierrez S, Silla-Martinez JM, \& Gabaldon T (2009) trimAl: a tool for automated alignment trimming in large-scale phylogenetic analyses. Bioinformatics 25(15):1972-1973.

107. Price MN, Dehal PS, \& Arkin AP (2009) FastTree: computing large minimum evolution trees with profiles instead of a distance matrix. Mol Biol Evol 26(7):1641-1650.

108. R-Development-Core-Team (2008) $R$ : A language and environment for statistical computing. (R Foundation for Statistical Computing, Vienna, Austria). 
872 109. Dray S, et al. (2017) adespatial: Multivariate Multiscale Spatial Analysis).

873 110. Paradis E, Claude J, \& Strimmer K (2004) APE: Analyses of Phylogenetics and $874 \quad$ Evolution in R language. Bioinformatics 20(2):289-290.

875 111. Wickham H (2009) ggplot2: Elegant Graphics for Data Analysis (Springer876 Verlag).

877 112. Chen J, et al. (2012) Associating microbiome composition with environmental 878 covariates using generalized UniFrac distances. Bioinformatics 28(16):21068792113.

880 113. Becker RA, Wilks AR, Brownrigg R, Minka TP, \& Deckmyn A (2017) maps: Draw Geographical Maps).

882 114. Gerritsen H (2014) mapplots: Data Visualisation on Maps).

883 115. Kembel SW, et al. (2010) Picante: R tools for integrating phylogenies and ecology. Bioinformatics 26(11):1463-1464.

885

116. Chase JM, Kraft NJB, Smith KG, Vellend M, \& Inouye BD (2011) Using null models to disentangle variation in community dissimilarity from variation in $\alpha$ diversity. Ecosphere 2(2):1-11.

887

888

117. Friedman J \& Alm EJ (2012) Inferring correlation networks from genomic survey data. PLoS Comput Biol 8(9):e1002687.

890 118. Watts SC, Ritchie SC, Inouye M, \& Holt KE (2018) FastSpar: Rapid and scalable correlation estimation for compositional data. bioRxiv.

119. Shannon P, et al. (2003) Cytoscape: a software environment for integrated models of biomolecular interaction networks. Genome Res 13(11):2498-2504.

120. Csardi G \& Nepusz T (2006) The igraph software package for complex network 
899

\section{Prokaryotes —Picoeukaryotes}

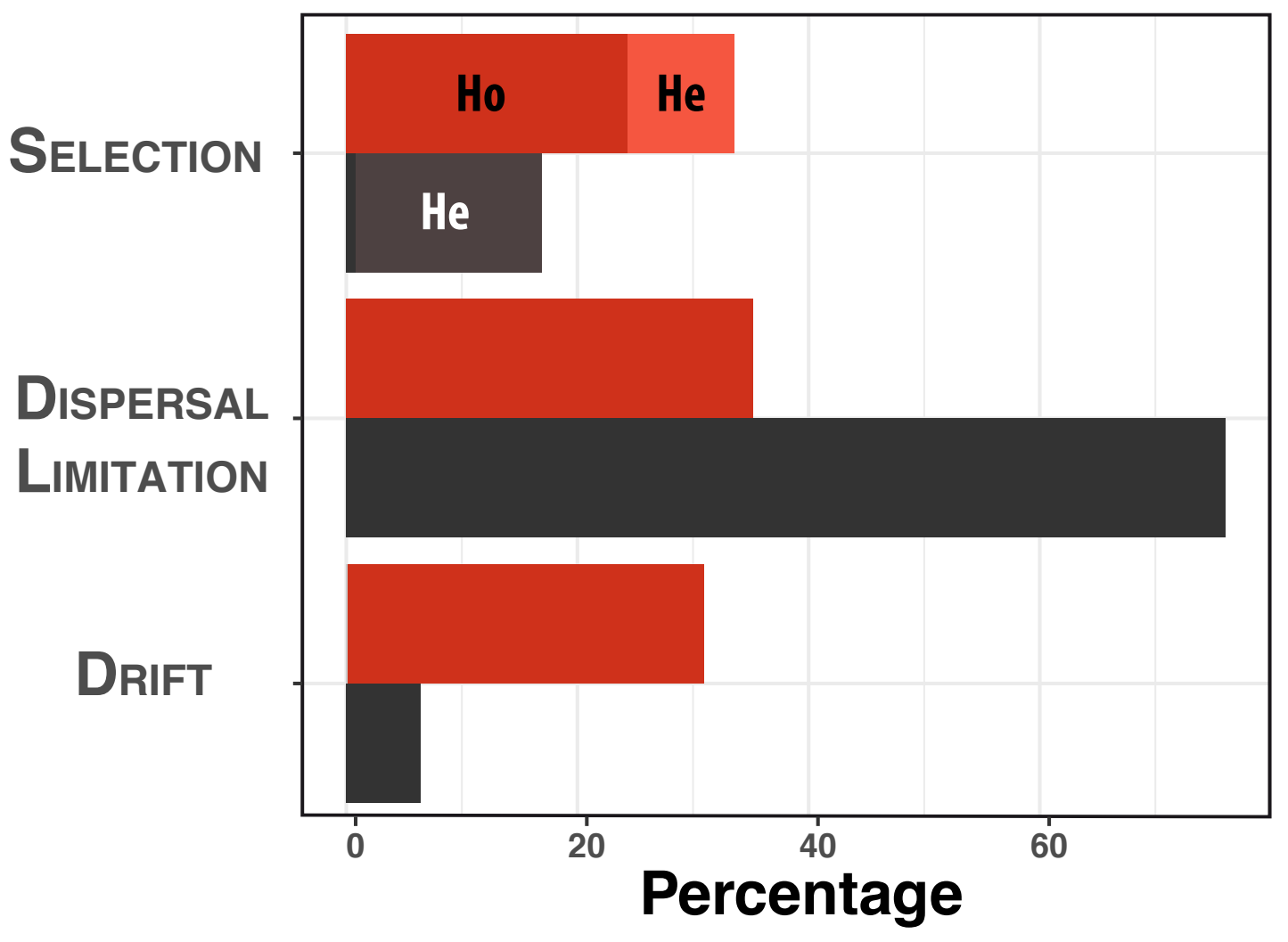

Figure 1. Relative importance of the processes shaping the tropical and subtropical

902 sunlit-ocean microbiome. Percentage of the spatial turnover governed by different

903 processes in prokaryotes and picoeukaryotes. $\mathrm{Ho}=$ Homogeneous selection, $\mathrm{He}=$

904 Heterogeneous selection (light red and light black).

905 


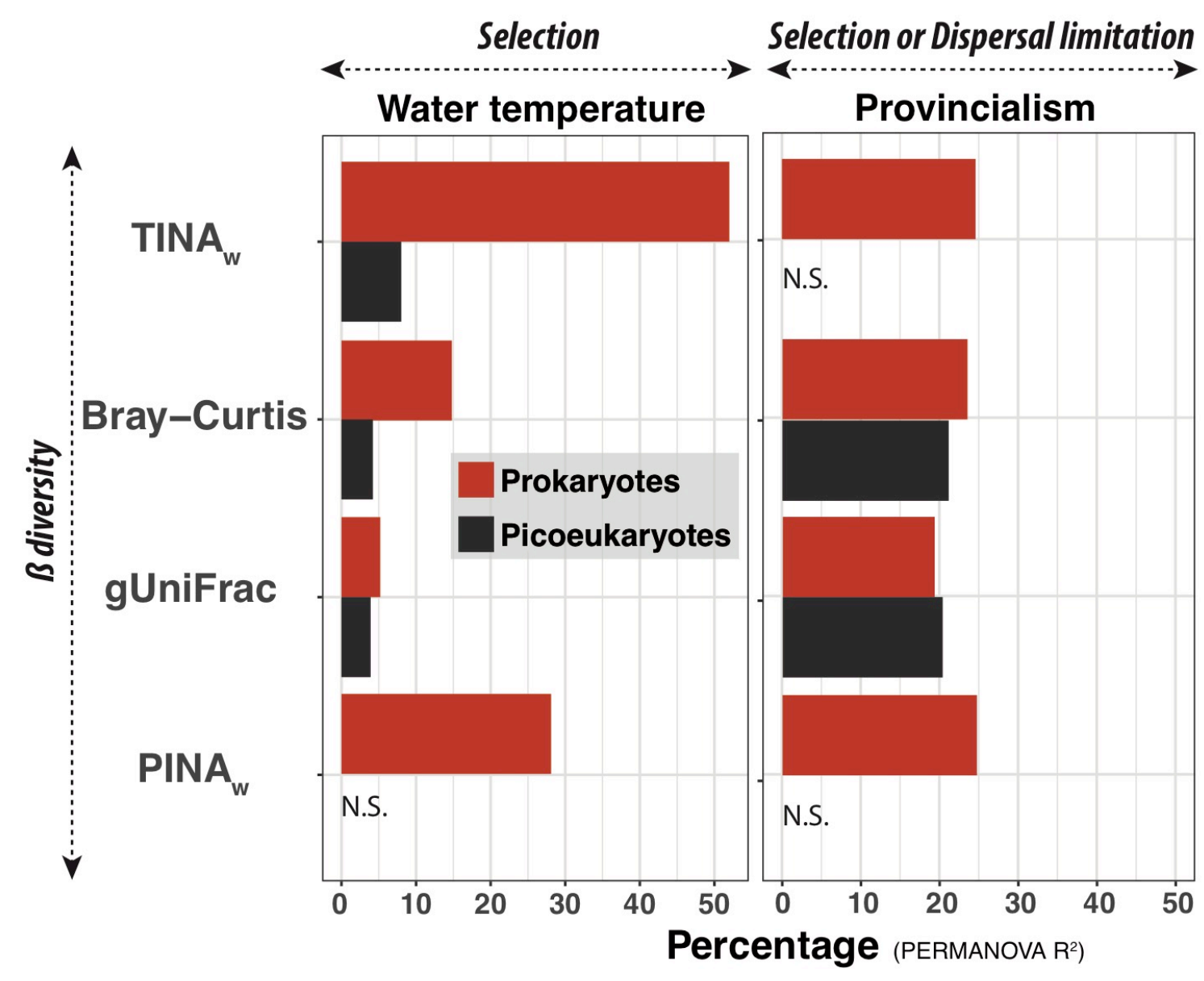

907 Figure 2. Water Temperature and Provincialism were the most important

908 variables explaining the structure of the tropical and subtropical surface-ocean

909 microbiome. Percentage of variance in picoeukaryotic and prokaryotic community

910 composition (PERMANOVA R ${ }^{2}$ ) explained by Water Temperature and Longhurst

911 Provinces when using different $\beta$-diversity metrics. Figure based on the Meta-119

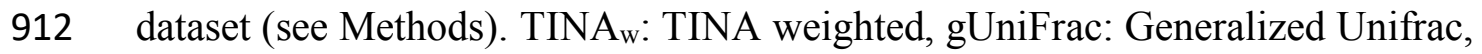

913 PINAw: PINA weighted. N.S. = Non-Significant. Note that TINA $_{\mathrm{w}}$ captures a

914 significantly higher proportion of community variance than Bray-Curtis in prokaryotes. 

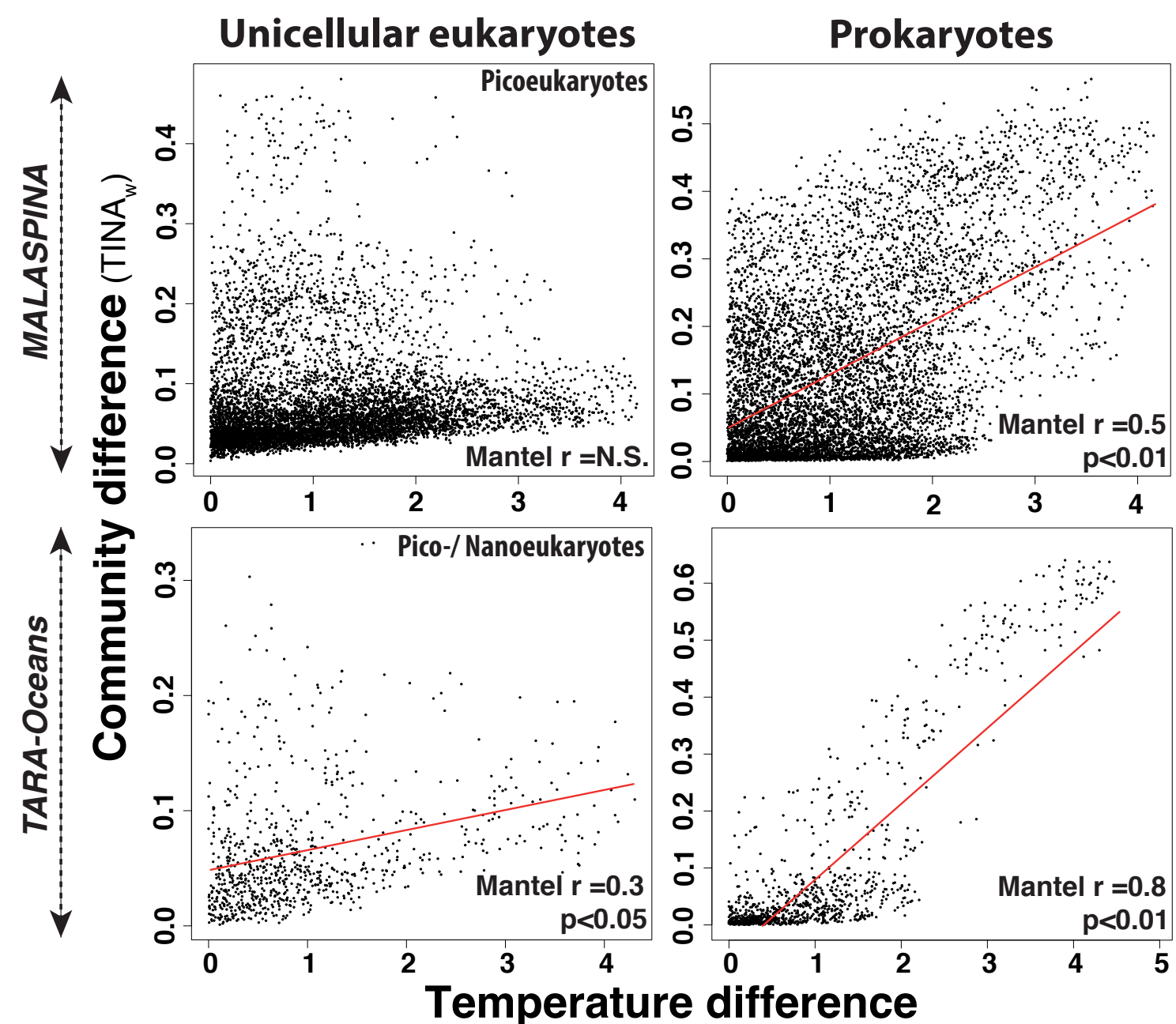

920

Figure 3. Temperature-driven selection affects species co-occurrences in

921 prokaryotes but not in small unicellular eukaryotes. Community differences (Tina-

922 weighted dissimilarities) vs. temperature differences (Euclidean distances) for both

923 small unicellular eukaryotes and prokaryotes sampled during the Malaspina and TARA

924 Oceans expeditions. NB: While only picoeukaryotes were contemplated in Malaspina

925 (cell sizes $<3 \mu \mathrm{m}$ ), TARA Oceans data included pico- and nanoeukaryotes (cell sizes $<5$

$926 \mu \mathrm{m})$. Pico- and nanoeukaryotes from both expeditions (left panels) displayed low or no

927 correlations between TINA $_{\mathrm{w}}$ distances and temperature differences (Note Mantel test

928 results in the figures). On the contrary, prokaryotes (right panels) had high to moderate 
929 correlations between TINA $_{w}$ distances and temperature differences. The regression line

930 is shown in red (Malaspina microbial eukaryotes N.S., Malaspina Prokaryotes $\mathrm{R}^{2}=0.3$,

931 TARA microbial eukaryotes $\mathrm{R}^{2}=0.1$, TARA Prokaryotes $\mathrm{R}^{2}=0.7 ; \mathrm{p}<0.05$ ).

932 




Figure 4. Spatial patterns likely manifesting differential dispersal or strong compared against its immediate predecessor. In Panels A and B, the size of each bubble represents the Bray-Curtis dissimilarity between a given community and the community

944 sampled previously. Blue squares in Panels A and B represent the stations where

945 pairwise $\beta$-diversity displayed abrupt changes (Bray Curtis values $>0.8$ for

946 picoeukaryotes and $>0.7$ for prokaryotes). Abrupt changes coincided in a total of 11 out

947 of 14 stations for both picoeukaryotes and prokaryotes, while one station presented

948 marked changes for picoeukaryotes and two for prokaryotes. Panel C summarizes the

949 sequential Bray-Curtis values for prokaryotes and picoeukaryotes [Means were 
950 significantly different between domains (Wilcoxon text, $\mathrm{p}<0.05$ )]. Panel D shows the 36

951 stations featuring a comparatively large contribution to the overall $\beta$-diversity (Local

952 Contribution to Beta Diversity (40); $\mathrm{p}<0.05$ ).

953

954 


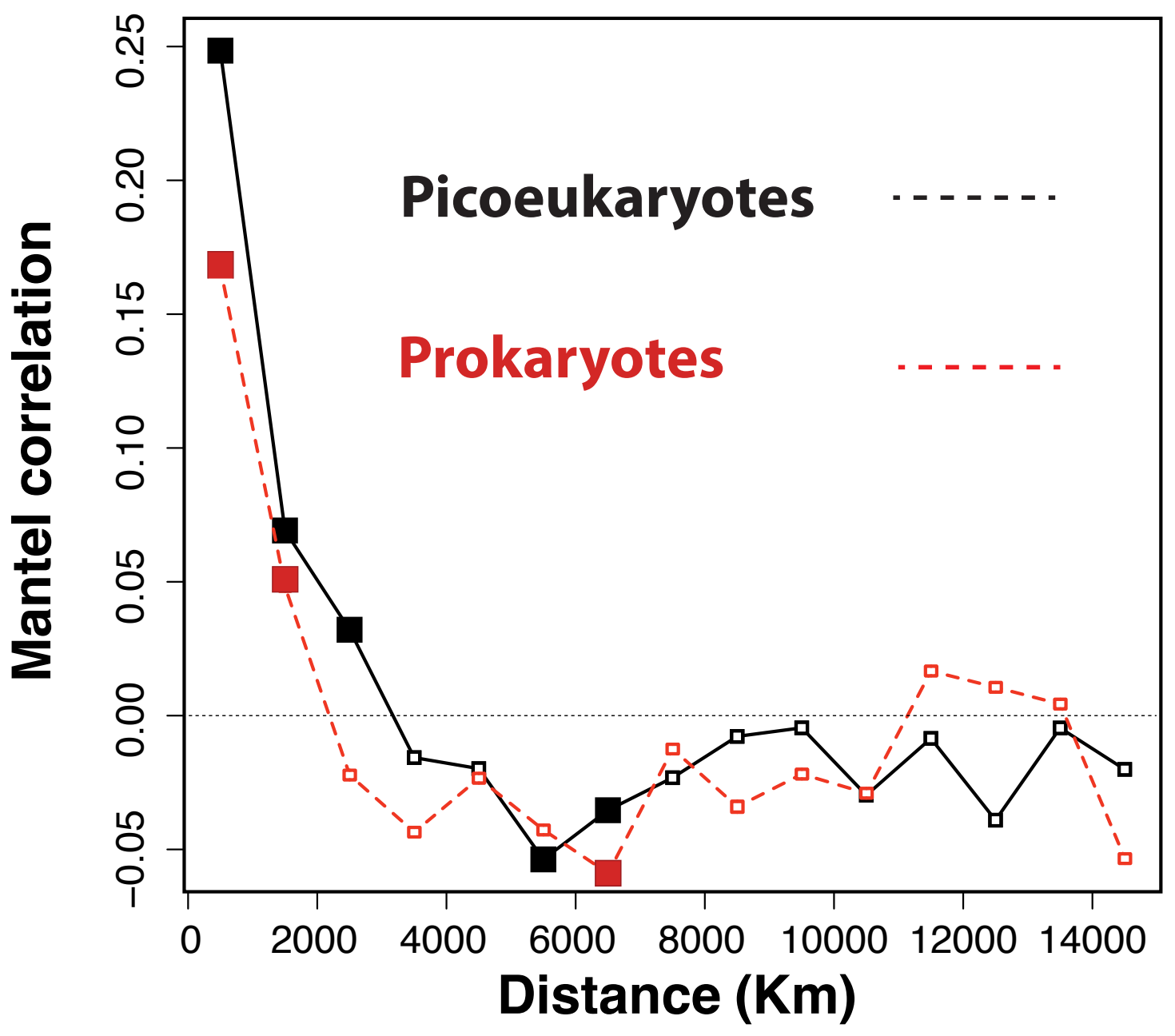

Figure 5. Decrease in community similarity with distance (distance decay). Mantel

$9611,000 \mathrm{~km}$ for both picoeukaryotes and prokaryotes. Coloured squares indicate

962 statistically significant correlations $(p<0.05)$. Note that $\beta$-diversity in picoeukaryotes

963 displayed positive correlations with increasing distances up to $\sim 3,000 \mathrm{~km}$, while

964 prokaryotes had positive correlations with distances up to $\sim 2,000 \mathrm{~km}$. Correlations

965 tended to be smaller in prokaryotes than in picoeukaryotes, indicating smaller distance

966 decay in the former compared to the latter. 